\title{
Use of Geospatial Information and Technologies in Understanding the COVID-19 Pandemic in Canada: Examples and Critical Discussion
}

\author{
David J. Coleman and Prashant Shukle
}

\begin{abstract}
Despite the very uneven distribution and intensity of the spread of COVID-19 in different regions - and considering highly decentralised responsibilities for health care, and shared federal and provincial leadership for national emergency response coordination - Canada has been able to ensure a reasonably effective and well-coordinated response. All Canadian provinces and territories now have data driven dashboards or geospatial tools that highlight the incidence of COVID-19 in their respective jurisdictions. After a number of early independent development initiatives sponsored by different provinces, a nationally-endorsed, geospatially-enabled exposure notification app for cellphones was publicly released at the end of July. While supportive of the overall efforts under difficult circumstances, the authors suggest that specific responses could be improved if proactive work is done to share technology and data within the framework of national pandemic or disaster plans. As well, it would be valuable to ensure that the geospatial tools employed and data collected continue to be used as the public-sector focus shifts from pandemic response to economic recovery. Strong leadership and high levels of both national and international cooperation will be required to address these improvements.
\end{abstract}

\subsection{Introduction}

As of early September 2020, the spread of COVID-19 still varies significantly across Canada. Some provinces such as British Columbia, Alberta, Ontario and especially Quebec experienced significantly higher impacts whereas other provinces and territories such as Manitoba, New Brunswick and Newfoundland \& Labrador experienced much lower levels of social and economic disruption. Despite this highly asymmetrical national spread of a global pandemic, highly decentralised responsibilities for health care, and shared federal and provincial leadership for national emergency response coordination - Canada has been able to ensure a reasonably effective and well-coordinated response. Governance and a long history of cross-jurisdictional collaboration have been critical success factors for Canada and reliance on data and technology were key enablers.

This chapter provides a very brief introduction to how they were deployed in Canada's COVID-19 pandemic. After providing selected examples of technology-level responses that were employed, the authors highlight early "hits and misses" which can be observed at this stage of the pandemic in Canada. The authors conclude with a look to the future in terms of opportunities for further development and cooperation. 


\subsection{Context}

\subsubsection{History and extent of the COVID-19 Outbreak in Canada}

Figure 29.1 is a political map showing Canada's provinces and territories. Detailed timelines of events surrounding the COVID-19 outbreak in Canada may be found at Public Health Canada [1] and the Canadian Press [2]. Very early in 2020, as information became available, Canadian efforts moved from a pragmatic "wait and see" approach to one of increased proactivity. By January 25, Canada's first case of COVID-19 was declared. Canada's Public Health Agency activated its Health Operations Centre and triggered Canada's Federal/ Provincial/ Territorial Public Health Response Plan for Biological Events [3]. Thousands of families were affected in the months to come: Prime Minister Trudeau announced on March 12 that he himself was self-isolating as his wife had tested positive for COVID 19. By September 6, 2020, almost 133,000 cases of the virus and 9145 deaths had been confirmed nationally (Public Health Canada, 2020b). Relative numbers of cases varied widely across the country, with three provinces in particular (Québec, Alberta and Ontario) accounting for a disproportionately high rate of those cases (Figure 29.2).

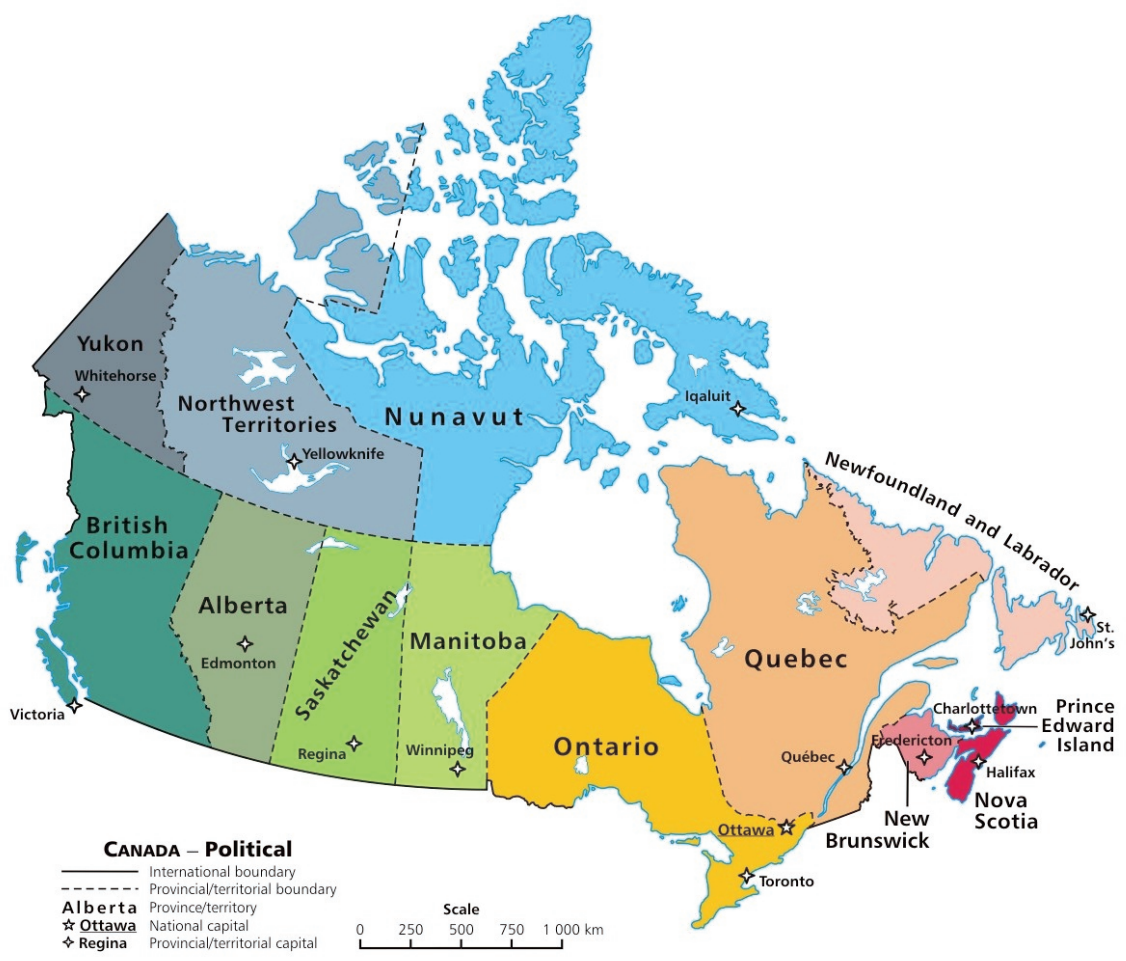

\section{FIGURE 29.1}

Map of Canada's Provinces and Territories [4] 


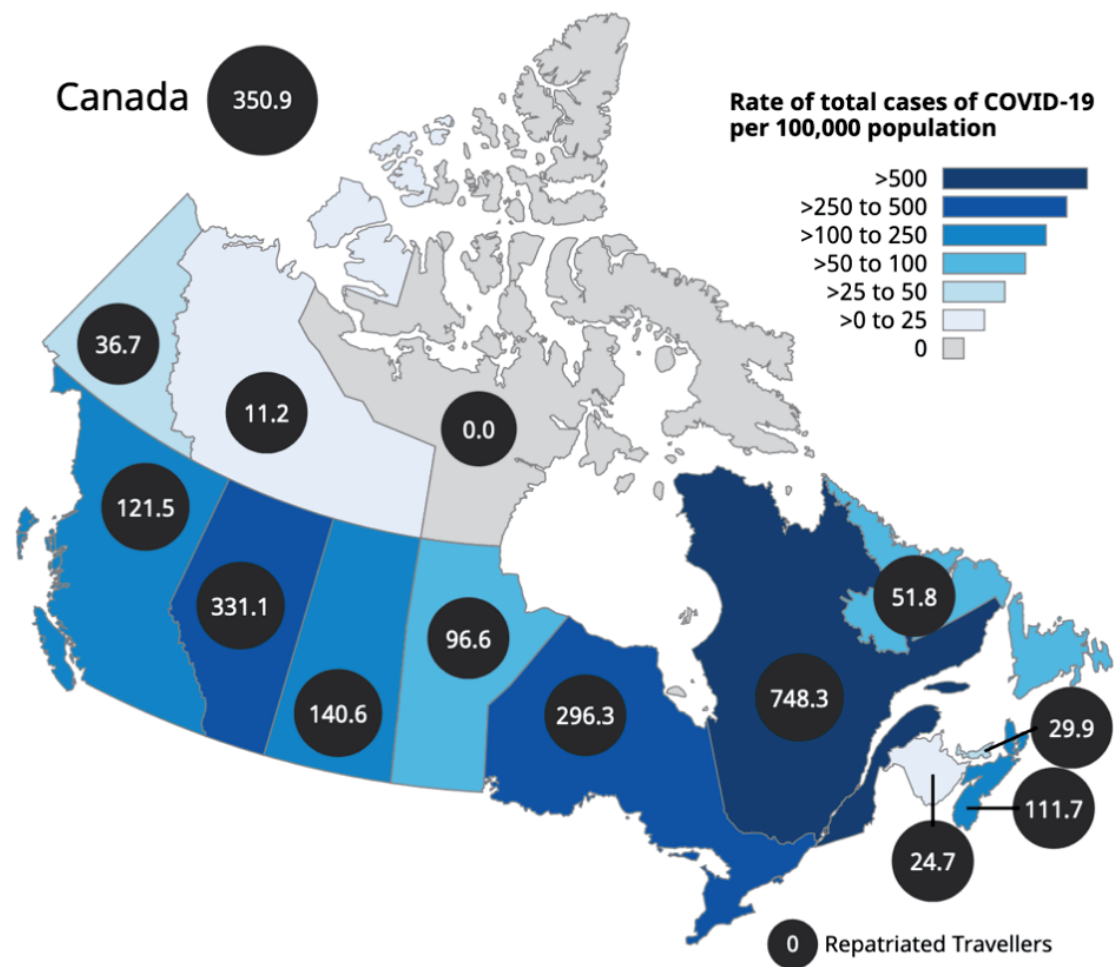

FIGURE 29.2

Rate of Confirmed Cases of COVID-19 per 100,000 population by Province as of September 6, 2020 [5]

No single factor accounts for the differences in the relative rate of cases in different provinces. Clearly the effects were greatest in large metropolitan centres, but that is only part of the picture. The high number of outbreaks in (especially) Nursing Homes and Seniors Care residences accounted for many of the cases found even in more sparsely populated provinces like Nova Scotia. Similarly, cities with busy international airports typically had higher incidence rates than those found elsewhere.

\subsubsection{Federal/Provincial Issues and Reactions}

Constitutionally, while Canada's federal government provides nation-wide leadership and coordination across all 14 jurisdictions in a national emergency (such as a global pandemic), each provincial government is responsible for adapting and implementing its own regional or local specific emergency management plan. Federal authorities are also required to provide 2 -way communication with all provinces and territories when coordinating the influx of returning citizens and international travellers who are exiting or entering the country at the more than 140 land border crossings, 14 international airports, and over 550 port facilities that play a strategic role in Canada's goods and services supply chain.

Approximately $80 \%$ of Canadians live within 160 kilometres miles of the Canada - United States border. The other $20 \%$ of the population resides in the remaining land mass that stretches a further 5300 kilometres north. If there was ever a crisis requiring an understanding of location and its relation to human health - this was it. 


\subsection{Institutional and Technical Responses}

Interestingly, the first capabilities to which Canadians turned were not geospatial in nature. Rather, governments at all levels relied upon institutional responsiveness and the reach of television, radio, social media, and newspapers. Depending on real-time events coupled with legacy economic and social influences in each province, each provincial government responded to their specific regional and local realities.

Communication strategies leveraged the power of a captive audience and the fact that $93 \%$ of Canadian adults are reached by television each week. To an impressive and unusual extent, federal, provincial and even (in major cities) municipal governments aligned to provide messaging that was overwhelmingly consistent, uniform, and readily available. Data initially focussed on the numbers of cases, the spread of the disease and the ongoing provision of expert advice and opinion. From March through May 2020, each provincial premier hosted daily news conferences that were televised on national and local media, usually following the Prime Minister of Canada who conducted his daily briefing for all Canadians. In the same sessions, expert senior public health authorities provided extensive information concerning the impacts, spread, and efforts at addressing COVID-19 in every federal, provincial and territorial jurisdiction.

Through different media channels, Canadians were urged strongly and consistently to: follow specific distancing and masking protocols, adopt telework arrangements, stay quarantined, and minimise exposure to each other by staying at home unless required. Given a tendency towards deference to authority and trust in governments, Canadians overwhelmingly adopted stringent health protocols.

\subsection{1 "Dashboard" Services}

To minimise speculation and conspiracy theorizing, all major political leaders in Canada threw their support behind expert management of the crisis. All governments established COVID-19 specific websites with extensive information and data about the impacts and preventative measures. For example, the Government of Canada released "Get Updates on COVID-19" a web-based email service that provides subscribers with critical information related to the pandemic. Developed by Health Canada, the Canadian Digital Service and Service Canada, the service ensured that those Canadians who subscribed would get authoritative and institutionally valid content through the Government of Canada website. Another application "ArriveCan", developed by the Canadian Border Services Agency and the Public Health Agency of Canada, allowed digitization of returning traveller information to help manage 14-day isolation information periods, including identification of whether the travellers have quarantine accommodations.

Baranovskiy et al. [6] offer an excellent early discussion of the difficulties in accessing, and organizing Canadian public datasets in order to create easy to understand thematic maps and charts that track the spread of COVID across the country. With "Made-in-Canada" geospatial solutions being initially slow off the mark, global substitutes were employed by national and local media outlets. For example, the John Hopkins Coronavirus Resource Center ${ }^{1}$ provided a very effective dashboard highlighting global cases, detailed U.S. caseloads, and other visualisations such as timelines and critical trends such as mortality rates. Another exceptional visualisation tool used by Canadian sources came from the Financial Times ${ }^{2}$, whose coronavirus data was used to illustrate not just the vectors and incidence of COVID-19 but also the economic impacts and shocks of the pandemic on global and national economies. Finally, the "COVID-19 Case Data Explorer" developed and maintained by Esri Canada [7] offers a very detailed up-to-date statistics at national, provincial and, in some cases, local health district levels.

It was not until May 2020 when the Government of Canada unveiled its first geospatially enabled dashboard which resulted from a shared collaboration between the Public Health Agency of Canada,

\footnotetext{
${ }^{1}$ See https://coronavirus.jhu.edu/map.html

${ }^{2}$ See https://on.ft.com/3d32Y1Z
} 
Statistics Canada, and Natural Resources Canada ${ }^{3}$. The dashboard, like that provided by Johns Hopkins, was powered by ESRI GIS capabilities and drew upon Government of Canada data and technology. Added visualisations included COVID in the world, a situational awareness dashboard visualising provincial and territorial data and other economic and demographic indicators ${ }^{4}$. All Canadian provinces and territories now have data driven dashboards or geospatial tools that highlight the incidence of COVID-19 in their respective jurisdictions.

\subsubsection{Geospatial Monitoring and Analysis - Contact Tracing}

Contact tracing is "...the process of identifying, assessing, and managing people who have been exposed to a disease to prevent onward transmission" [8]. Public health agencies have long employed the tracing the contacts of infected individuals (and then their contacts in turn) in order to inform policies and practices which reduce infections in the population. Digital contact tracing today relies on positioning and tracking capabilities of (primarily) mobile personal devices in order to determine contact between a given infected patient and the device user.

Different countries have adopted different technical approaches and sometimes conflicting guiding philosophies to digital contact tracing. There is considerable debate on the user population-base requirements necessary to ensure effective contact tracing as well as the attendant privacy and security implications to individual citizens using contact tracing apps $[9,10]$.

Exposure notification applications rely on the same basic technology but purports to offer greater privacy protection to individual users [11]. Exposure notification apps notify you if you have been near someone who later tested positive for COVID-19. By comparison, contact tracing apps let people log their location and then share it (voluntarily or involuntarily) with public health authorities.

Through April-May 2020, Canada saw multiple efforts carried out by independent provincial governments in Alberta, Newfoundland \& Labrador, Ontario, Manitoba, Saskatchewan and New Brunswick to either procure or develop in-house their own apps to support "exposure notification" [12].

Most of these initiatives intended to incorporate Bluetooth technology provided by Apple and Google. However, to avoid duplication and confusion, those companies made it clear they preferred supporting one nationally-endorsed app rather than many different regional ones [13]. The federally-funded COVID Alert app development project was led by the Canadian Digital Service and being undertaken in cooperation with partners from the Ontario Provincial Government and with help from volunteers from the commercial technology firm Shopify [14]. While originally expected to be rolled out by July 1, 2020, unexpected issues in refinement and testing delayed the national app's introduction until July 31, 2020 - and even then its reception was mixed [15].

From an institutional perspective, widespread public and commercial interest in COVID-19 related contact tracing apps quickly sparked concerns from government Privacy Commissioners and citizen interest groups alike across Canada. Two institutional responses provided early and very useful contributions to this debate. In April 2020, the Office of the Privacy Commissioner of Canada issued an assessment framework [16] intended to "... assist government institutions faced with responding to the COVID-19 crisis, and help organizations subject to federal privacy laws understand their privacy-related obligations during the pandemic". This framework was followed the next month with a more extensive Joint Statement by Federal, Provincial and Territorial Privacy Commissioners articulating their shared position on privacy principles for contact tracing and exposure notification [17].

Given the engagement of the Privacy Commissioner of Canada and other key geospatial departments such as Natural Resources Canada - concerns for individual privacy and protection directed the final application design to utilise Bluetooth to exchange random codes with nearby phones that also have the app involved. If someone within the proximate areas scanned by the phones later tests positive for COVID-19 they are to entire a key or code into the app. If any individual has spent more than 15 minutes and less than the mandated 2 metres apart from the

\footnotetext{
${ }^{3}$ See https://health-infobase. canada.ca/covid-19/

${ }^{4}$ https://health-infobase.canada.ca/covid-19/visual-data-gallery/
} 
infected individual they will be notified. It should be noted that this app will not provide location, names, addresses, places, the time of contact or any health information to anonymise and protect the health information of Canadians.

\subsection{Discussion}

The "purpose-built" development of the new Canadian Government geospatial dashboard devoted specifically to COVID-19 tracking is notable for two reasons. First, it took well over 3 months for the relevant data and tools on the new dashboard to be configured and launched publicly. At the same time, the Government of Canada already had its own Federal Geospatial Platform [18] as an enterprise wide geospatial capability - one that had been highly touted as one of the Government of Canada's most successful data and technology initiatives. In the authors' opinion, this lack of responsiveness and subsequent duplication requires further study. Second, the time lag in preparing Canadian data dashboards suggests a gap in the institutionally driven pandemic Response Plan. Although this Plan had clearly undergone significant consultations and trial runs beforehand, it suggests the need for greater advance planning to reduce this time lag for data loading in the future.

As well, the authors look forward to seeing reports on the use of geospatially-enabled dashboards and exposure notification for operational modelling purposes in Canada through different stages of the pandemic. A significant amount of relevant health data on the spread and incidence of the disease may already be available to public authorities in geospatial and analysis-ready formats, but its veracity is still unclear. While we appreciate there are real and significant difficulties in planning and conducting such analyses effectively [19, 20], critical assessments of examples and lessons learned in this regard will be extremely valuable to the international community. Similarly, there has been no documentation yet on the impact and use of that geospatial data for economic and health recovery in Canada. Geospatially-enabled analysis of supply chains, detailed economic analyses at local levels, and sentiment analyses of citizens in hardest hit areas are all within the grasp of the technology of today. The authors look forward to seeing - hopefully soon - work undertaken in this regard as well.

\subsection{Towards the Future}

Geography - in particular, the very small number of densely-populated large metropolitan areas in relation to its vast area - has played a key role in Canada's relative success in containing the COVD-19 pandemic. Still, if the number of confirmed cases and mortality statistics are any indication, Canadian responses to the COVID-19 pandemic have been relatively well managed and of the "middle of the pack" variety. While new apps have been developed, websites built, and a variety of data disseminated to citizens, the first priority was clearly to use mass media to communicate information and expert advice in order to ensure that citizens were protected from the effects of COVID-19 and that the general population benefitted from the mitigation of any spread.

That said, the issues highlighted earlier do present significant institutional and technologydevelopment opportunities in Canada.

First, most jurisdictions could respond more quickly if proactive work is done to share technology and data within the framework of national pandemic or disaster plans. Critical questions highlighting which data are essential to report and how should form a critical element of a clear data strategy in each plan.

Second, these pandemic plans should also focus on the recovery - it will be essential to understand how the health recovery is progressing alongside an economic recovery, and key social, 
environmental and health indicators from national to local perspective would be critical to a well managed and staged set of economic and social policy interventions.

Finally, as we move further away from any pandemic or crisis, there is a clear need to ensure that tools and data enabling pandemic response are transformed and sustainably utilised for the next set of crises that depend on geospatial data.

Global readiness depends on long term vigilance. Leadership and international cooperation will be required in the GGIM academic community and well beyond in order to ensure all nations are able to attain the necessary levels of technical and data readiness.

\section{References}

[1] Public Health Canada. History section, Coronavirus disease (COVID-19): Outbreak update. 2020. https: //www.canada.ca/en/public-health/services/diseases/2019-novel-coronavirus-infection.html Last accessed 30 June 2020.

[2] Canadian Press. A timeline of events in Canada's fight against COVID-19. 2020. https://www.thestar. com/news/canada/2020/06/18/a-timeline-of-events-in-canadas-fight-against-covid-19.html Last accessed 22 July 2020.

[3] Public Health Canada. Federal/Provincial/Territorial Public Health Response Plan for Biological Events. 2018. https://www.canada.ca/en/public-health/services/emergency-preparedness/public-health-responseplan-biological-events.html Last accessed 22 July 2020.

[4] E.P. Anthony. Map of Canada - by E Pluribus Anthony, transferred to Wikimedia Commons by Kaveh (log), optimised by Andrew pmk. - Own work, Public Domain. 2006. URL https://commons.wikimedia.org/w/index. php?curid=844714. Last accessed 7 September 2020.

[5] Public Health Canada. Current Situation section, Coronavirus disease (COVID-19): Outbreak update. 2020. Map Generated Courtesy of Public Health Canada Web Mapping Site https://health-infobase.canada.ca/ covid-19/dashboard/.

[6] Peter Baranovskiy, Ken Coates, and Carin Holroyd. "Designing COVID-19 Data Tools". Part of the JSGS Policy Briefs COVID-19 Series: From Crisis to Recovery, Johnson Shoyama Graduate School of Public Policy, University of Saskatchewan, Saskatoon, SK, Canada. 2020. https://www.schoolofpublicpolicy.sk.ca/ research/publications/policy-brief/designing-covid-19-data-tools.php Last accessed 7 September 2020.

[7] Esri Canada. COVID-19 Case Data Explorer. 2020. https://bit.1y/34zmuPN Last accessed 7 September 2020.

[8] World Health Organization. Contact tracing on the context of COVID-19. Interim Guidance Document, Reference No. WHO/2019-nCoV/Contact_Tracing/2020.1. 2020. https://www. who.int/publications/i/item/ contact-tracing-in-the-context-of-covid-19 Last accessed 30 June 2020.

[9] Robert A. Kleinman and Colin Merkel. Digital contact tracing for COVID-19. CMAJ, 192(24):E653-E656, 2020. doi: https://doi.org/10.1503/cmaj.200922.

[10] Jeffrey Kahn and Johns Hopkins Project on Ethics and Governance of Digital Contact Tracing Technologies. Digital Contact Tracing for Pandemic Response: Ethics and Governance Guidance. Johns Hopkins University Press, 2020. https://muse.jhu.edu/book/75831 Last accessed 30 June 2020.

[11] Caroline Haskins. Apple and Google's Coronavirus Tech Won't Actually Do Contact Tracing. Here's Why Exposure Notification Is Different. Buzzfeed News, 20 May 2020. https://www.buzzfeednews.com/article/ carolinehaskins1/what-are-exposure-notifications-contact-tracing-how-are Last accessed 30 July 2020.

[12] Ruth Promislow, Michael Whitt, and Stephen Burns. COVID-19 and Contact-Tracing Apps in Canada. Blog entry. Bennett Jones LLP, 2020. https://www.bennettjones.com/Blogs-Section/COVID-19-and-ContactTracing-Apps-in-Canada Last accessed 22 June 2020.

[13] Catharine Tunney. Ottawa looking to endorse 1 coronavirus contact tracing app for the whole nation. Online news article, Canadian Broadcasting Corporation, Ottawa, Canada, 2020. https://www.cbc.ca/ news/politics/trudeau-app-contact-tracing-1.5580184 Last accessed 22 May 2020.

[14] Government of Canada. Download COVID Alert Today. 2020. https://www.canada.ca/en/public-health/ services/diseases/coronavirus-disease-covid-19/covid-alert.html Last accessed 7 September 2020.

[15] Thomas Daigle. Misconceptions persist about effectiveness and privacy of Canada's COVID Alert app. $C B C$ News, Toronto, Canada, 2020. https://www.cbc.ca/news/technology/covid-19-alert-app-myths-privacy-1. 5684089 Last accessed 7 September 2020. 
[16] Privacy Commissioner. A Framework for the Government of Canada to Assess Privacy-Impactful Initiatives in Response to COVID-19. Office of the Privacy Commissioner of Canada, 2020. https://www.priv.gc.ca/en/ privacy-topics/health-genetic-and-other-body-information/health-emergencies/fw_covid/ Last accessed 30 June 2020 .

[17] Privacy Commissioner. "Supporting public health, building public trust: Privacy principles for contact tracing and similar apps". A Joint Statement by Federal, Provincial and Territorial Privacy Commissioners. Office of the Privacy Commissioner of Canada, 2020. https://www.priv.gc.ca/en/opc-news/speeches/2020/s-d_ 20200507/ Last accessed 30 June 2020.

[18] Government of Canada. Federal Geospatial Platform. 2020. https://www.nrcan.gc.ca/science-data/scienceresearch/earth-sciences/geomatics/canadas-spatial-data-infrastruct/geospatial-communities-canadace/federal-geospatial-platform/11031 Last accessed 22 July 2020.

[19] Chenghu Zhou, Fenzhen Su, Tao Pei, An Zhang, Yunyan Du, Bin Luo, Zhidong Cao, Juanle Wang, Wen Yuan, Yunqiang Zhu, Ci Song, Jie Chen, Jun Xu, Fujia Li, Ting Ma, Lili Jiang, Fengqin Yan, Jiawei Yi, Yunfeng Hu, Yilan Liao, and Han Xiao. COVID-19: Challenges to GIS with Big Data. Geography and Sustainability, 1(1):77 - 87, 2020. ISSN 2666-6839. doi: https://doi.org/10.1016/j.geosus.2020.03.005. URL http://www.sciencedirect.com/science/article/pii/S2666683920300092.

[20] Kenneth Field. Mapping coronavirus, responsibly. ArcGIS Blog, 2020. https://www.esri.com/arcgis-blog/ products/product/mapping/mapping-coronavirus-responsibly/ Last accessed 30 June 2020. 